\title{
Diagnóstico de la gestión de la construcción e implementación de la constructabilidad en empresas de obras civiles
}

\section{Diagnosis of construction management and constructability implementation in construction}

\author{
Zulay Giménez Palavicini*1, Carlos Suárez Isea*
}

* Universidad Centroccidental Lisandro Alvarado, VEN EZU ELA

Resumen

Fecha de recepción: 20/ 01/2008 Fecha de aceptación: 20/ 03/ 2008 PAG. $04-17$

\begin{abstract}
Este artículo muestra los resultados de una investigación, en la cual se realizó un diagnóstico de las empresas de edificación que operan en la ciudad de Barquisimeto, Venezuela; para conocer el grado de aplicación de los conceptos de constructabilidad, reconocer las barreras para la implementación de dichos conceptos y la disposición de la alta gerencia de adoptar la metodología; para luego realizar una propuesta de los cambios pertinentes para la consolidación del programa de constructabilidad en la(s) empresa(s). Igualmente se estudió la realidad interna de las empresas en cuanto a aspectos como: programación y planificación, dificultades en obra, abastecimiento, características del personal, uso de optimización y facilidades tecnológicas, que forman parte de la gestión cotidiana de la construcción. Los principales resultados son el alto grado de desconocimiento tanto del término "constructabilidad" como de sus beneficios de implementación, así como también gran disposición de las empresas en adoptar la metodología para la optimización de sus procesos.
\end{abstract}

Palabras Clave: Constructabilidad, gestión de la construcción, implementación, planificación, experiencia

Abstract

This paper presents research results diagnosing processes and performance of leading construction firms in Barquisimeto, Venezuela. Studied topics include: constructability application concepts, recognition of constructability barriers, top management recommendations to adopt the methodology, decision making process to lead program consolidation through change management. In addition, several internal aspects relative to construction firm management were analyzed, such as: scheduling and planning, construction difficulties, procurement, personnel characteristics, optimization process and technology implementation.

Keywords: Constructability, management of the construction, implementation, planning, experience

\section{Introducción}

El proceso de constructabilidad ha sido estudiado por el CII (Instituto de la Industria de la Construcción, según sus siglas en inglés) desde aproximadamente el año 1984, y ha generado conceptos que aportan mejoras en todas las etapas de la gestión de proyectos constructivos, así como también una metodología para la implementación exitosa de la constructabilidad en las empresas motivadas a hacerlo. Los países que han empleado la constructabilidad han logrado buenos resultados en el ámbito constructivo y han mejorado los procesos en las diferentes etapas del proyecto, cumpliendo con la programación de obras estipuladas sin retrasos no previstos, entre otros avances.

La constructabilidad no es más que un programa de mejoramiento continuo en el área de la construcción y tiene como finalidad integrar el diseño con la construcción como tal, incorporando la experiencia y el conocimiento constructivo en etapas tempranas como la planificación, diseño, abastecimiento y otras, haciendo más factible su ejecución, mas "construible" el proyecto.

\footnotetext{
${ }^{1}$ Autor de correspondencia / Corresponding author:

Universidad Centroccidental Lisandro Alvarado. Prolongación Av. La Salle, antigua escuela ferroviaria. Venezuela.

Teléfono: 00582512592157

E-mail: zulaygimenez@ucla.edu.ve
} 
La constructabilidad se emplea en países desarrollados, contratándose consultores privados para que revisen los diseños con una óptica constructiva, buscando la factibilidad de desarrollarlos en el menor tiempo y costo posibles. Así mismo, cada proyecto debería ser elaborado bajo una política de calidad que permita cuantificar el comportamiento de la mano de obra, minimizar accidentes, mejorar los procesos en las diferentes etapas de la construcción, utilizar el tiempo y recursos justos sin desperdicio, cumplir con la programación de obras estipulada sin retrasos no previstos, integrar adecuadamente abastecimiento, ejecución, uso de herramientas y maquinarias, horarios y mano de obra capacitada; y una interrelación más clara entre proyectistas y constructores, así como también una eficiente comunicación entre la gerencia y los trabajadores.

El Cll publicó en 1993 el material: Constructability implementation guide; en éste se muestra un juego completo de 19 herramientas para la implementación de los programas de constructabilidad. Está destinada a propietarios, diseñadores y personal de la construcción con particular énfasis en los propietarios. Presenta 17 conceptos de constructabilidad además de las barreras más comunes en la implementación de dichos programas.

El objetivo de esta investigación, realizada en el marco de un trabajo de grado para obtener el título de M. Sc. en gerencia de la construcción, es el de mejorar la gestión de la construcción a través de la implementación de la constructabilidad en empresas de obras civiles de la ciudad de Barquisimeto. Para ello, se realizó en primer lugar, un diagnóstico de la situación de las empresas constructoras seleccionadas de la ciudad, en cuanto a su comportamiento con respecto a las diversas actividades realizadas en las distintas áreas de la gestión de la construcción (abastecimiento, programación, planificación, entre otros) y el uso de los conceptos de constructabilidad, así como también respecto a la presencia de barreras importantes podrían inhibir la implementación efectiva de programas de mejoramiento de la gestión de la construcción en las empresas, aspectos que serán mostrados parcialmente en este artículo.

\section{Diseño de la investigación}

Se realiza un diagnóstico de tipo transversal, ya que se recolectan datos en un solo momento, en un tiempo único; su propósito es describir variables y analizar su incidencia en un momento dado. Se califica como una investigación de campo, ya que se buscó la información en forma directa a través de cuestionarios a 38 empresas de obras civiles de la ciudad de Barquisimeto, seleccionadas del universo conformado por las empresas afiliadas a la "Cámara de la Construcción del Estado Lara" de acuerdo a sus características en cuanto a tamaño y obras importantes ejecutadas dentro del sector. Para esto se diseñó un instrumento acorde con los objetivos planteados y los conceptos evaluados de constructabilidad.

El número estimado de empresas para dicha muestra, se basó en registros de la Cámara de la Construcción del Estado Lara (http://www.ccl.com.ve), el cual tiene 340 agremiados entre empresas constructoras, proveedores de insumos y servicios para la construcción, entidades bancarias y agencias inmobiliarias. De los 340 agremiados, aproximadamente 220 son empresas constructoras, que es realmente el ámbito que interesa en esta investigación. Sin embargo, aplicar 220 cuestionarios en localidades distintas resulta engorroso, poco práctico, caro y sobre todo innecesario. Es por ello que se elige una muestra, en principio de 30 empresas. Este valor se toma, partiendo de Walpole y Myers, 2000; quienes afirman "para muestras de tamaño $n \geq 30$, sin importar la forma de la mayoría de las poblaciones, la teoría muestral garantiza buenos resultados".

Tomando en consideración, que no todas las empresas colaborarían con el llenado de cuestionarios de tipo académico, se seleccionaron 45 empresas constructoras de las 220 iniciales, con la posibilidad de que si 15 de ellas no colaboraran (33\%), igualmente la muestra sería de 30 empresas. De las 45 empresas seleccionadas, 38 colaboraron y llenaron por completo el cuestionario dado, solo 7 de ellas no los respondieron, por diversas razones, que no viene al caso mencionar.

En esta investigación, aún siendo cuantitativa, no se ha formulado ningún tipo de hipótesis, ya que Hernández Sampieri (2004) afirma "Las investigaciones cuantitativas, cuyo método es el deductivo sí formulan hipótesis, siempre y cuando se defina desde el inicio que su alcance será correlacional o explicativo, o en caso de un estudio descriptivo, que intente pronosticar una cifra o un hecho. "En este caso, no se pretendió pronosticar un hecho o una cifra en sí, sino simplemente describir las características de las empresas para llegar a conclusiones coherentes y extender los resultados a un universo más amplio.

A continuación se muestra (Tabla $\mathrm{N}$ ㅇ) la información general de la muestra con respecto al alcance geográfico, tipo de servicios prestados por las empresas y su tipo de contratación en cuanto al ente: si es público o privado. 
Tabla 1. Información general de la muestra. Alcance

\begin{tabular}{|c|c|c|c|}
\hline \multicolumn{4}{|c|}{ Alcance de la empresa } \\
\hline \multicolumn{4}{|c|}{ Ciudad (es) donde opera: } \\
\hline \multicolumn{3}{|c|}{ Barquisimeto solamente } & $66 \%$ \\
\hline \multicolumn{3}{|c|}{ Barquisimeto y otras ciudades del Estado Lara } & $5 \%$ \\
\hline \multicolumn{3}{|c|}{ Barquisimeto y otras ciudades de la Región Centroccidental } & $16 \%$ \\
\hline \multicolumn{3}{|c|}{ Barquisimeto y otras ciudades del país } & $13 \%$ \\
\hline \multicolumn{4}{|c|}{ Tipo de servicios prestados por la empresa: } \\
\hline \multicolumn{3}{|c|}{ Proyectos de Diseño y Cálculos } & $53 \%$ \\
\hline \multicolumn{3}{|c|}{ Promoción Inmobiliaria } & $39 \%$ \\
\hline \multicolumn{3}{|c|}{ Construcción de Edificaciones } & $84 \%$ \\
\hline \multicolumn{3}{|c|}{ Construcción de Obras Viales } & $26 \%$ \\
\hline \multicolumn{3}{|c|}{ Construcción de Obras Sanitarias e Hidráulicas } & $39 \%$ \\
\hline \multicolumn{3}{|c|}{ Otros } & $32 \%$ \\
\hline \multicolumn{4}{|c|}{ Relación de contratación pública/privada } \\
\hline \multicolumn{2}{|c|}{ Empresas } & \multicolumn{2}{|c|}{ Tipo de contratación } \\
\hline Número & Porcentaje & Pública & privada \\
\hline 15 & $39 \%$ & $0 \%$ & $100 \%$ \\
\hline 2 & $5 \%$ & $5 \%$ & $95 \%$ \\
\hline 3 & $8 \%$ & $10 \%$ & $90 \%$ \\
\hline 3 & $8 \%$ & $20 \%$ & $80 \%$ \\
\hline 1 & $3 \%$ & $30 \%$ & $70 \%$ \\
\hline 2 & $5 \%$ & $70 \%$ & $30 \%$ \\
\hline 2 & $5 \%$ & $80 \%$ & $20 \%$ \\
\hline 3 & $8 \%$ & $90 \%$ & $10 \%$ \\
\hline 7 & $18 \%$ & $100 \%$ & $0 \%$ \\
\hline Fuente Propia & & & \\
\hline
\end{tabular}

\subsection{Técnica de recolección de datos}

Se elaboró un cuestionario con base en una escala de Likert (Hernández, 2004), con el cual se midió la capacidad de la empresa de utilizar conceptos de constructabilidad; así como también conocer si los han implementado o no y qué disposición tendría de adoptarlos. Además se conoce la existencia de los síntomas de las barreras más comunes para la implementación de la constructabilidad. Esta escala consta de 5 valoraciones, en la que la respuesta óptima representa el número valor. (Ver anexo 1)

El cuestionario consta de 10 secciones sin embargo en este artículo solo se mostrarán los resultados de 6 de estas (Ver anexo 2), las cuales sirvieron para realizar el diagnóstico de la empresa en diferentes aspectos:

1. identificación de barreras de implementación

2. programación de obras

3. consideraciones y previsiones en general
4. optimización y facilidades tecnológicas

5. conocimiento del programa de la constructabilidad

6. disposición de la implementación de dicho programa

También se observa la opinión de dichas empresas sobre la situación actual de la construcción, en cuanto a las dificultades presentadas en obra. Este instrumento se diseñó con base en los 17 conceptos básicos de Constructabilidad, (Serpell, 2002; CII, 1993), y de los síntomas de las barreras mas comunes para la implementación de un programa de constructabilidad (CII, 1993); que tuvieran relación con los aspectos mencionados anteriormente.

Aún cuando el cuestionario era anónimo, es decir, no se debía colocar el cargo ni el nombre del entrevistado, éste debía poseer un alto cargo dentro de la empresa o de la obra (dueño, director, gerente, líder de obra, etc.). 


\section{Análisis de los resultados}

3.1 Barreras para la implementación de la constructabilidad
En esta sección se observan claramente los síntomas mas comunes de las barreras para la implementación de la constructabilidad en las empresas. En el anexo 3, se muestra la relación de las preguntas del cuestionario con los diversos síntomas de las barreras más comunes. Las once barreras consideradas en esta primera sección se pueden clasificar en tres grupos de acuerdo a su frecuencia:

El primer grupo representa los síntomas mas frecuentes, por lo tanto debe darse una importancia mayor en cuanto a la implementación de "rompebarreras" adecuados. Se observa que el síntoma mas frecuente es la falta de documentación de errores y sus posibles correcciones, esto realmente es muy común entre las empresas constructoras y se evidencia en estos resultados. El documentar los errores cometidos es de vital importancia ya que si éstos no se evalúan y se documentan de manera práctica y sencilla, con facilidad se pueden volver a cometer. El segundo y el tercer síntoma se relacionan mucho, ya que la falta de visión de benchmarking, es decir, el no compararse con los mejores en el área para mejorar, sugiere una falta de estrategia, la cual es una de las primeras medidas que se deben utilizar al entrar en el mundo de la globalización y la competencia del mercado. "Las empresas deben formular estrategias en cuanto al alcance, ventajas competitivas empleadas, recursos y sinergias, que no es mas que las complementariedades que se pueden establecer entre la estrategia y las demás áreas de la organización" (Villalba, 1999). En cuanto al cuarto síntoma (El personal de construcción no revisa el proyecto durante el proceso de diseño), es imprescindible que la experiencia constructiva revise el diseño, antes que culmine la secuencia para evitar modificaciones posteriores en las obras, que resultan mucho mas costosas que en los planos.

El segundo grupo representa los síntomas con una frecuencia media, es decir, debe dársele importancia, luego de haber resuelto los inconvenientes que representan el primer grupo. Al haber problemas de comunicación entre áreas y falta de simplicidad en la manera de explicar los proyectos, se generan muchos errores que pueden incrementar considerablemente los costos; estos son evitables con una estrategia de reuniones y formatos establecidos, entre otras tácticas. Esta afirmación se genera de un grupo de trabajo simultáneo en una de las empresas encuestadas. Igualmente, "Ios propietarios, en particular, pueden crear oportunidades para una comunicación más abierta mediante la selección adecuada de tipos de contrato, la ejecución de los proyectos y los métodos de colaboración para realizar proyectos, y al exigir un proceso formal que incorpora la experiencia de la construcción de manera temprana en el proyecto"(Pocock, 2006). El personal de diseño generalmente tiene poco conocimiento del área de la construcción; esto puede deberse a muchas causas, que es importante averiguar en el ámbito de cada empresa por separado para establecer la(s) estrategia(s) a utilizar mas adecuada(s) según el caso. El no evaluar o evaluar de manera inadecuada los errores y problemas presentados en las obras, es un síntoma muy importante que se debe atacar; este se relaciona con el mas frecuente de la lista (falta de documentación de los mismos). Las empresas evalúan los errores verbal e informalmente, pero no llegan a registrarse 0 documentarse, por lo que además de perderse formalidad, se pierde efectividad en el tiempo. Son comunes también los últimos dos síntomas de este grupo: falta de motivación en la implementación de innovaciones y nuevas estrategias, esto tiene que ver con la usual "resistencia al cambio" "si esto lo hemos hecho así durante tantos años y siempre ha servido ¿por qué cambiarlo?". Este síntoma se puede romper con investigación exhaustiva de las innovaciones presentes en el mercado y la mejora (cuantificable) de las mismas en cuanto a rendimientos, productividad y rentabilidad.

El último grupo presenta los aspectos que las empresas controlan mejor, ya que los síntomas muestran un grado de ocurrencia muy bajo. Se refiere a los problemas de relaciones personales entre los empleados de la empresa y la complacencia con su estado actual. Esto quiere decir que aunque se presenten inconvenientes, los empleados interactúan de manera respetuosa y amable la mayoría del tiempo. Igualmente el empresariado en general considera que su empresa puede mejorar sustancialmente la eficacia, que es bien importante ya que esto siempre le va a permitir mejoras.

\subsection{Previsiones y consideraciones}

En el Figura № 2, se observan en qué etapa se consideran o prevén aspectos importantes del proyecto. Se considera óptimo, según la constructabilidad, el prever todos estos aspectos en las primeras etapas: planificación $\mathrm{y} / \mathrm{o}$ diseño. Por lo tanto se ordenaron los diferentes aspectos de acuerdo a su grado de previsión en estas primeras etapas. 


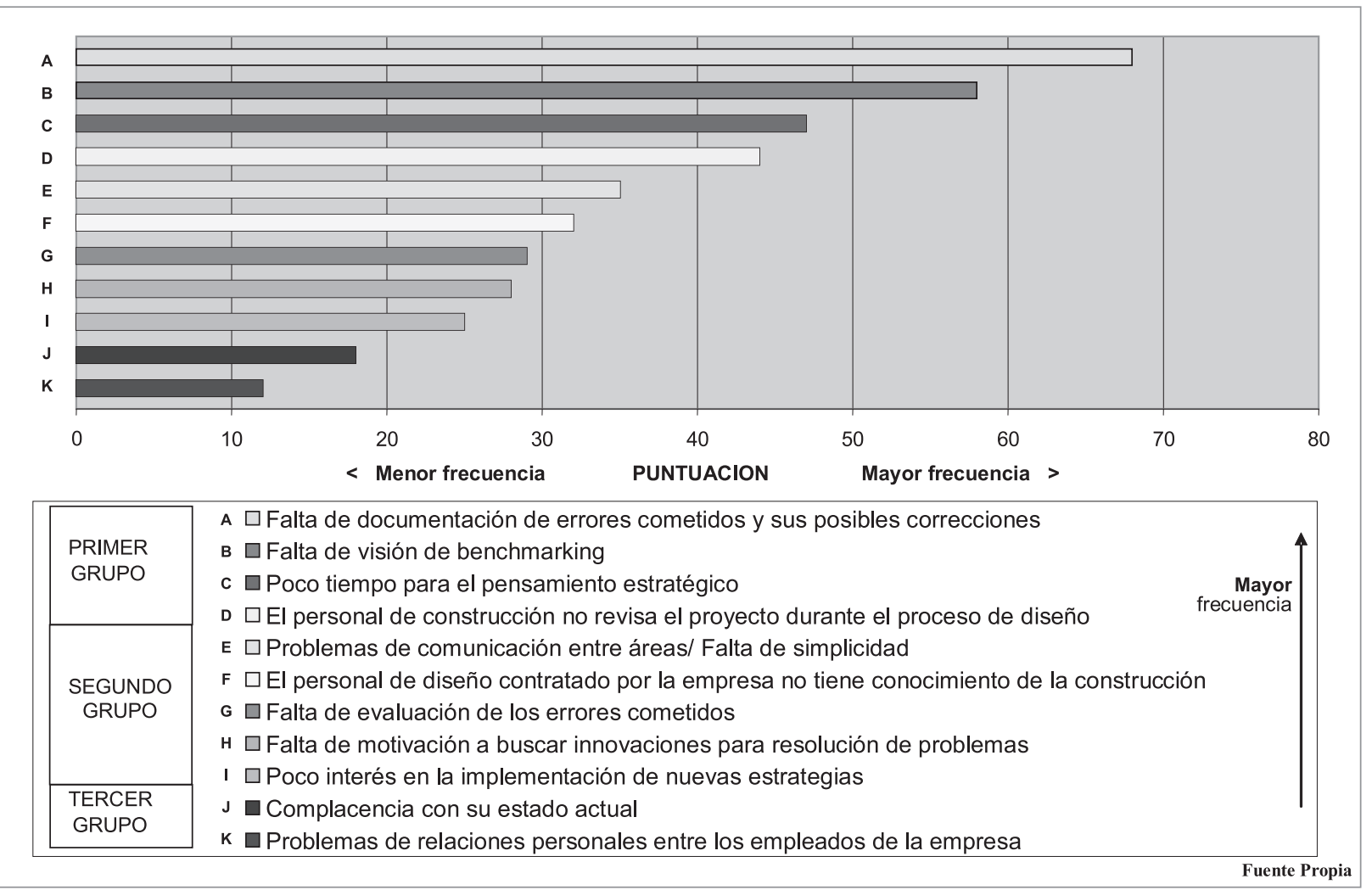

Figura 1. Síntomas de barreras según su frecuencia

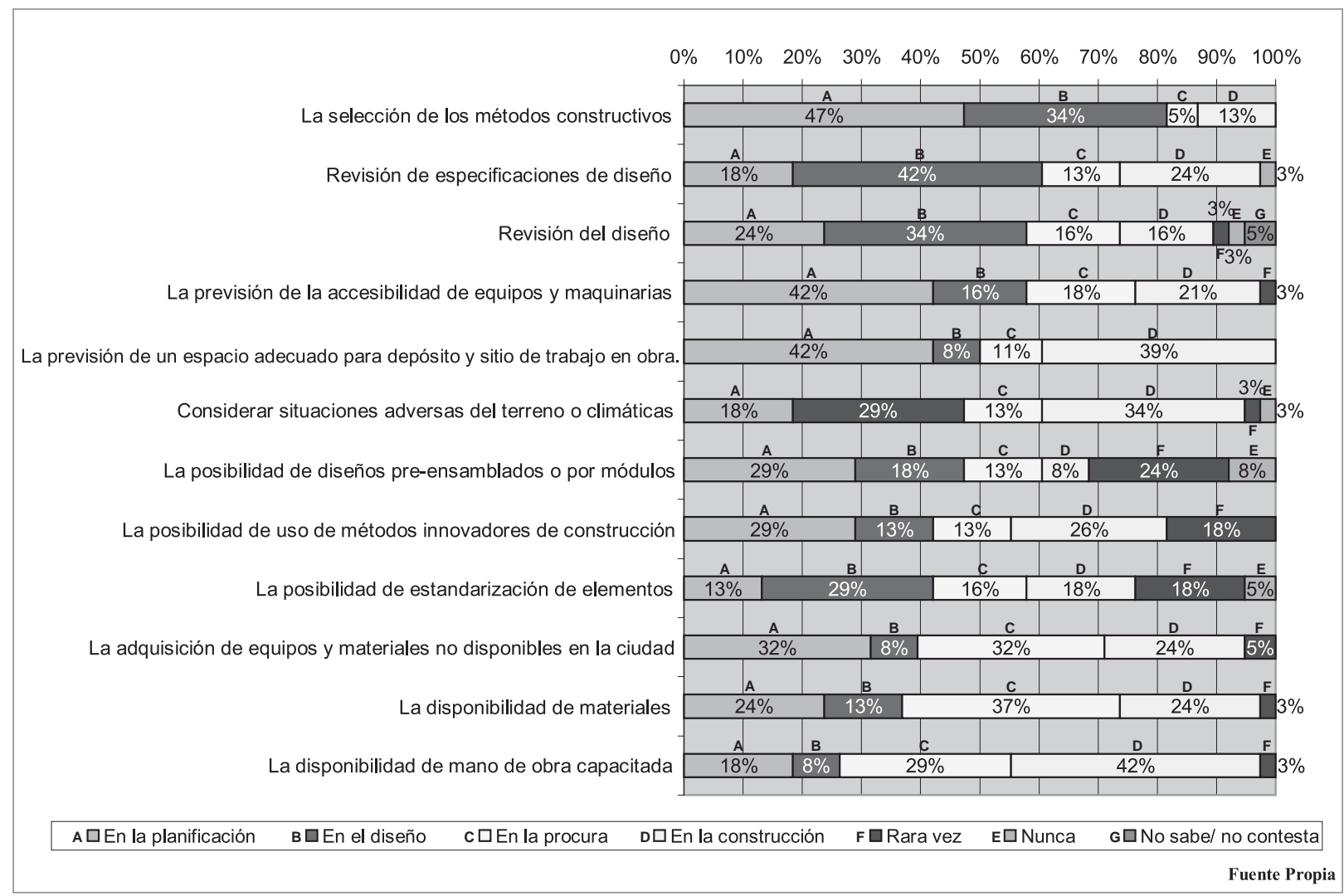

Figura 2. Previsiones y consideraciones 
Se podría decir que más del $50 \%$ consideran la selección de los métodos constructivos, revisión de las especificaciones y del diseño, así como la previsión de la accesibilidad de equipos y maquinarias en estas dos primeras etapas, lo que se considera positivo. Sin embargo, Ilama la atención el hecho de que el $11 \%$ de las empresas no sepan (o no contesten) si existe revisión del diseño $(5 \%)$, o que rara vez se realice ( $3 \%)$ o, lo que resulta grave, simplemente que no realicen dicha revisión del diseño $(3 \%)$. Aunque es un porcentaje bajo, la falta de revisión del diseño podría traer como consecuencia modificaciones en obra, que resultan generalmente en aumento en los costos y/o pérdida de rentabilidad del proyecto. Entre el 40 y el $50 \%$ de las empresas toman en consideración los siguientes 5 aspectos en estas primeras etapas de planificación y/o diseño: la previsión de un espacio adecuado y sitio de trabajo en obra, considerar situaciones adversas o climáticas, posibilidad de diseños pre-ensamblados, estandarización o uso de métodos innovadores de construcción. Sin embargo el 3\% de las empresas nunca consideran las condiciones climáticas adversas, lo que genera posiblemente modificaciones en la programación establecida.

Los aspectos menos considerados en estas etapas son la adquisición de equipos y materiales no disponibles en la ciudad y la disponibilidad de materiales y de mano de obra capacitada. Esto trae como consecuencia, y se verá en cuadros y gráficos posteriores, que los problemas de abastecimiento y de contratación del personal traen grandes dificultades en las obras que generan retrasos importantes. En la Figura 3 se muestra el divorcio existente entre el diseño y la construcción. Es preocupante que sólo el $18 \%$ de los diseños se respete de tal manera que se construya como fue previsto por el grupo de diseñadores. Es vital investigar las razones por las cuales las obras no se construyen exactamente igual al diseño original para determinar los correctivos necesarios a dicha problemática.

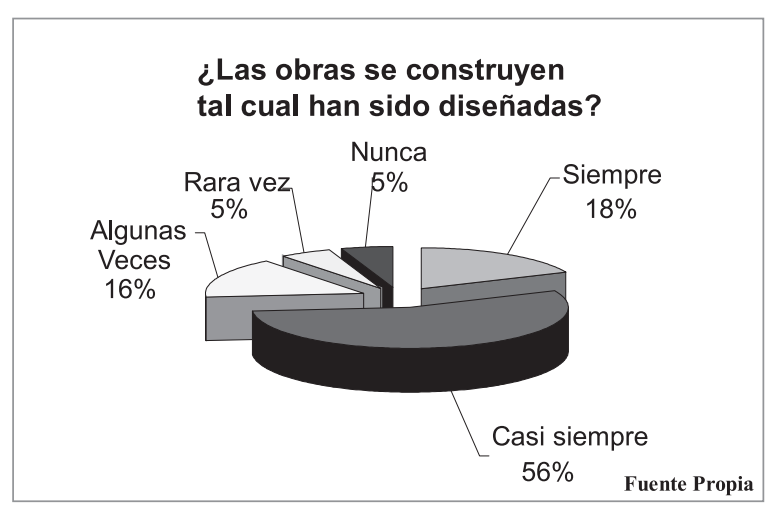

Figura 3. Divorcio existente entre el diseño y la construcción

\subsection{Programación de obras}

La Figura 4 muestra claramente que no todas las empresas realizan programación de sus obras. Sólo el 58\% de las empresas siempre la llevan a cabo. El cuestionamiento a consecuencia de esta realidad es conocer qué mecanismos utilizan las empresas en las obras que no realizan programación, para saber la duración total de la obra, la secuencia que van a llevar, cómo controlan las actividades de la obra, entre otras muchos aspectos.

Ninguna de las empresas cumple cabalmente la programación establecida. La mayoría de las empresas (55\%) respondió "casi siempre". El 16\% de las empresas presentan problemas con la programación establecida, al responder que nunca o rara vez se cumple la programación establecida. Es importante destacar que la programación de la obra puede no cumplirse al $100 \%$ por muchas razones tanto internas como externas. Aunque no era una pregunta del cuestionario, muchos empresarios manifestaron estas razones entre las cuales se podrían nombrar: (1)La realización poco realista del programa de obras, bien sea por lógica incorrecta, por duraciones sobreestimadas 0 subestimadas; (2) la no consideración ( o ligereza en las misma) de las condiciones climáticas; (3) porque se obviaron algunas actividades importantes que incidieron notablemente en la duración total del proyecto; (4) Por problemas con la disponibilidad de materiales; (5) Por problemas con la disponibilidad de personal o empresas contratistas, entre otras.

Con respecto a la diferencia entre la duración real y la propuesta, cabe destacar que es una pregunta distinta a la anterior, ya que es posible que siendo la duración real igual a la propuesta, las duraciones y secuencias de las actividades hayan variado, por ello la programación sería distinta a lo ejecutado realmente. Sólo en el $3 \%$ de las empresas coincide la duración real de la obra con la duración propuesta, el $26 \%$ estima que casi siempre coinciden, lo que representa que el $29 \%$ de las empresas son exitosas hasta cierto punto en cuanto al cálculo de la duración de la obra. 0 tro $26 \%$ de las empresas tienen muchos problemas con la determinación de la duración total de la obra debido a que nunca o rara vez coinciden con la duración real. El $45 \%$ se sitúa en un nivel intermedio (responde "a veces"). Es importante destacar que realizar la programación de una obra requiere de experiencia en construcción, manejo de tiempos reales, uso de estadísticas de tiempos de proyectos anteriores; aspectos que no todos los constructores dominan. 
El $68 \%$ de las empresas culminan en su totalidad las obras y un $24 \%$ de las empresas casi siempre. Sólo el $3 \%$ de las empresas consideró que "rara vez" culmina la obra en su totalidad. Sería interesante averiguar el estado de dichas obras inconclusas y las causas que llevaron a no culminarlas.

\subsection{Porcentaje de exceso en los costos}

En la Figura 5 se pueden observar aspectos interesantes, en primer lugar el $8 \%$ de las empresas realmente no conoce el porcentaje de excesos en los costos, mucho menos por las causas planteadas. Por otra parte, podemos observar tres tipos de empresas, 1) las que manejan hasta cierto punto los excesos en los costos en un rango establecido entre el 0 y el $10 \%$, representan el $41 \%$ de la muestra; 2 l) las empresas que tienen unos excesos en los costos entre el 11 y el $20 \%$, representan el $40 \%$ de las empresas, este margen implica pérdidas importantes en la rentabilidad de la misma, ya que las inversiones en el sector de la construcción son muy altas y el porcentaje de rentabilidad, hasta cierto punto, bajo con respecto a otros sectores productivos; y 3) empresas que no tienen dominio absoluto sobre los costos, ya que el exceso lo asumen por encima del 30\%, incluso puede llegar a ser del $75 \%$ (3\% de las empresas), esto implica una pérdida tan importante en montos de dinero en obra, que no toda empresa podría afrontar fracasos monetarios de este tipo.

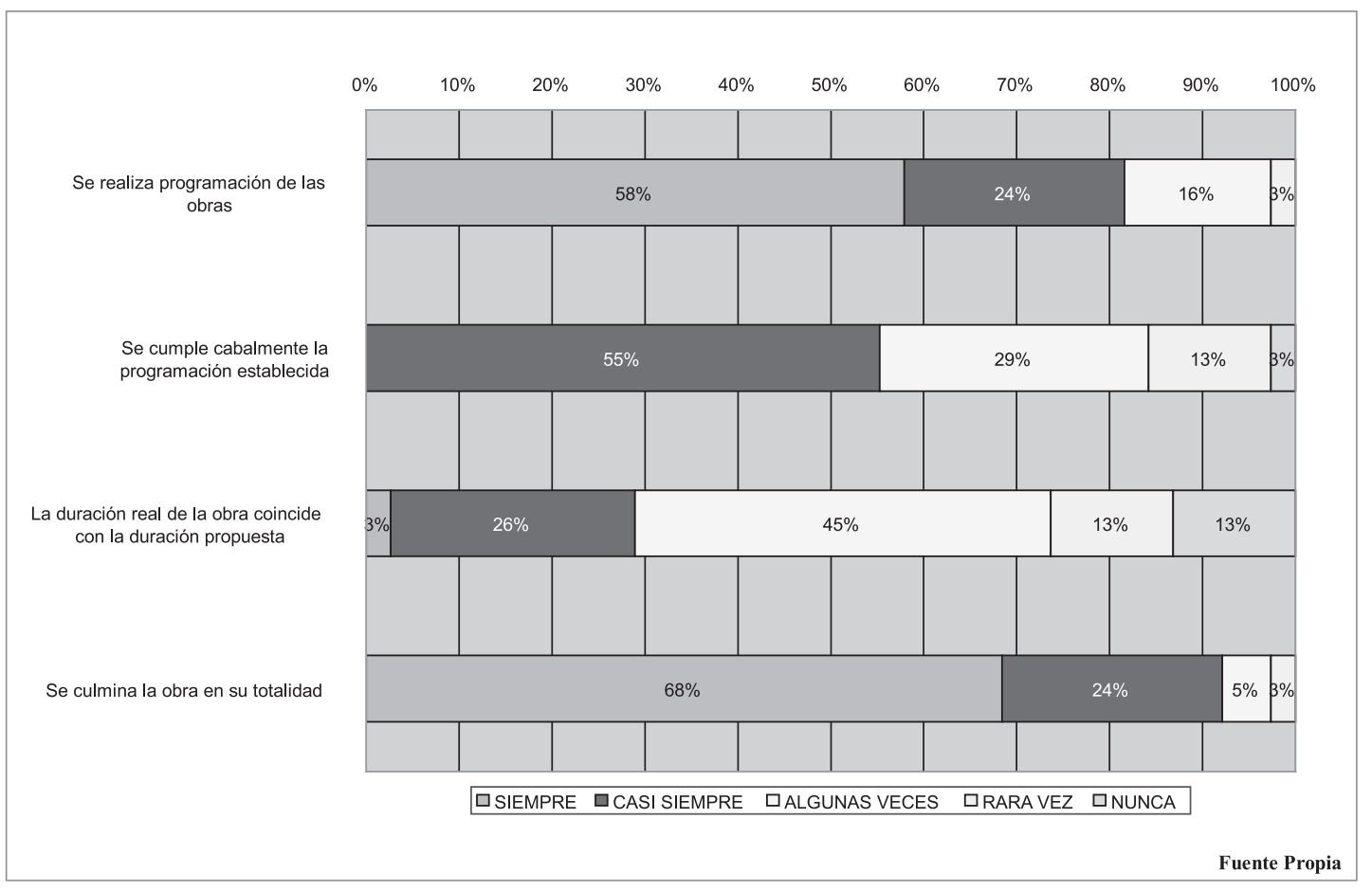

Figura 4. Programación de obras

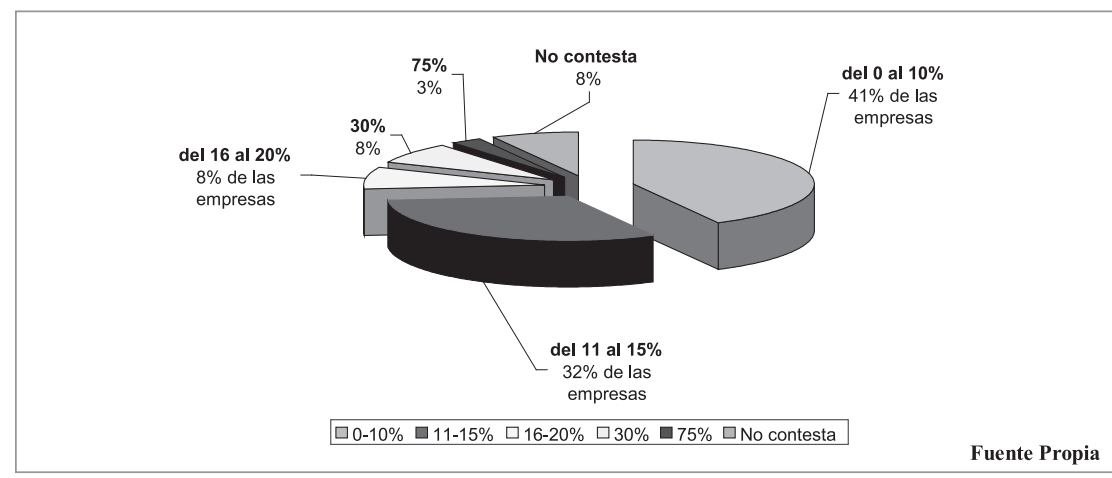

Figura 5. Porcentaje de excesos en los costos por conceptos de errores, retrasos u omisiones 
3.5 Dificultades en obra

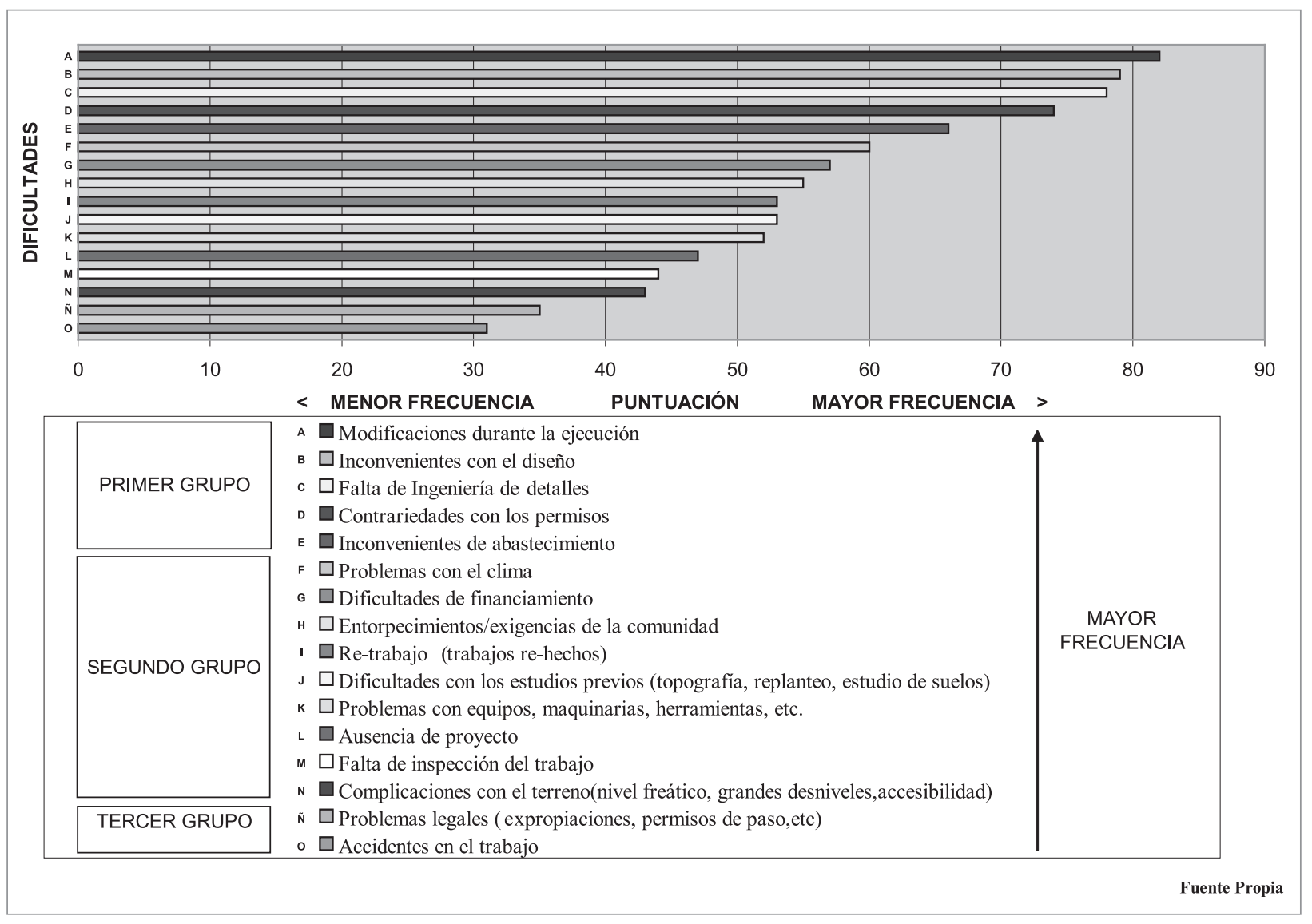

Figura 6. Dificultades en obras

La Figura 6 ha sido clasificado en tres grupos para su análisis: el primer grupo representa los aspectos que con mayor relevancia deben ser tratados, para minimizar sus consecuencias, sin descuidar desde luego las dificultades descritas en los grupos con menor frecuencia. En éste se encuentran los siguientes aspectos:

Las modificaciones durante la ejecución: (cualquier modificación, sea cual fuere debe realizarse en el proceso de diseño o de planificación de la ejecución, ya que incide de manera significativa tanto en los costos como en los tiempos);

Los inconvenientes de diseño y falta de ingeniería de detalles, pueden generar retrasos significativos. Rischmoller (2006), "Jornadas de Constructabilidad" realizadas el 27 de octubre de 2006 en nuestra ciudad, afirma que el $50 \%$ del tiempo del profesional de la construcción lo utiliza "buscando información no explícita en los planos", si dos de los aspectos mas frecuentes en nuestras empresas de la ciudad es que existen inconvenientes de diseño y falta de ingeniería de detalles, es posible que este porcentaje (del $50 \%$ ) se profundice en nuestros profesionales.

El cuarto ítem más común es "contrariedades con los permisos", para este aspecto se recomienda la figura de un gestor de permisos con el fin de que pueda resolver estos problemas con diligencia. Cabe destacar que generalmente los permisos son otorgados por distintos organismos, con procesos habitualmente engorrosos y son gestionados directamente por los arquitectos y diseñadores.

Los inconvenientes de procura, como aspecto común pueden ser consecuencia directa de la falta de previsión en este aspecto, tal como lo muestra la Figura 2, previsiones y consideraciones.

El segundo grupo en relación con la frecuencia plantea aspectos tanto del interior de la empresa como de influencia externa de la misma, representa el grupo de los aspectos no críticos, pero si importantes para ser tratados 
y previstos en su oportunidad. Se presentan a continuación: Problemas con el clima, y los entorpecimientos o exigencias de la comunidad, aunque son externos a la empresa, pueden influir de manera considerable en la ejecución de una obra, aún así se pueden prever o generar mecanismos adecuados para minimizar su incidencia en la misma.

Las dificultades de financiamiento, aunque no es un aspecto técnico o "constructivo" puede incluso paralizar una obra, por lo tanto se debe cuidar con sumo interés este aspecto.

Los trabajos re-hechos pueden ser consecuencia de otros aspectos presentes en esta misma lista.

Dificultades con los estudios previos, se recomienda una revisión exhaustiva de los mismos como requisito precedente a la entrega, así como también una metodología ya establecida para sistema de reuniones, formatos, revisiones y entrega formal del estudio. Estos estudios generalmente son realizados por profesionales $y$ empresas externas.

Problemas con equipos, maquinarias y herramientas, para evitarse deben no solamente preverse que se encuentren en el sitio cuando se necesiten sino también desarrollar dentro de la empresa una cultura de mantenimiento preventivo de los mismos.

Los últimos cinco aspectos, son los considerados como menos frecuentes, que sin embargo no deben descuidarse para que no presenten problemas serios a la empresa. Con respecto al ítem "Ausencia de proyecto", esta dificultad aunque con frecuencia relativamente baja, llama mucho la atención que ocurra.
Es importante destacar que el orden que arroja el gráfico es de la muestra de empresas encuestadas, es posible que una empresa en particular tenga un orden distinto al planteado, por lo tanto es vital para la empresa que desee mejorar la gestión de la construcción y erradicar o minimizar las dificultades en las obras, conocer su jerarquía propia para así comenzar por la mas frecuente. Esta lista es importante, ya que puede representar el punto de partida de cualquier plan de acción que quiera emprender una empresa al iniciarse en el mejoramiento de su gestión de la construcción.

\subsection{Programa de constructabilidad}

En cuanto al uso o aplicación de estos procesos, cabe destacar que cualquiera de ellos implica un cambio de cultura dentro de la empresa, es por ello, que aunque el interés de esta investigación se basa en la constructabilidad; se ve la importancia de que si una empresa aplica o está en proceso de aplicar la ingeniería del valor o la certificación ISO 9000:2000, ya ésta tiene un gran camino recorrido en el mejoramiento continuo y por lo tanto sería mas fácil la implementación de la constructabilidad en la misma.

Con respecto al conocimiento en sí del programa de constructabilidad, en el gráfico № 8 se observa el poco conocimiento (alrededor del 90\%) de las empresas de la ciudad con respecto al programa de constructabilidad, los beneficios de su implementación y la relación costobeneficio de la implementación de la constructabilidad en la empresa.

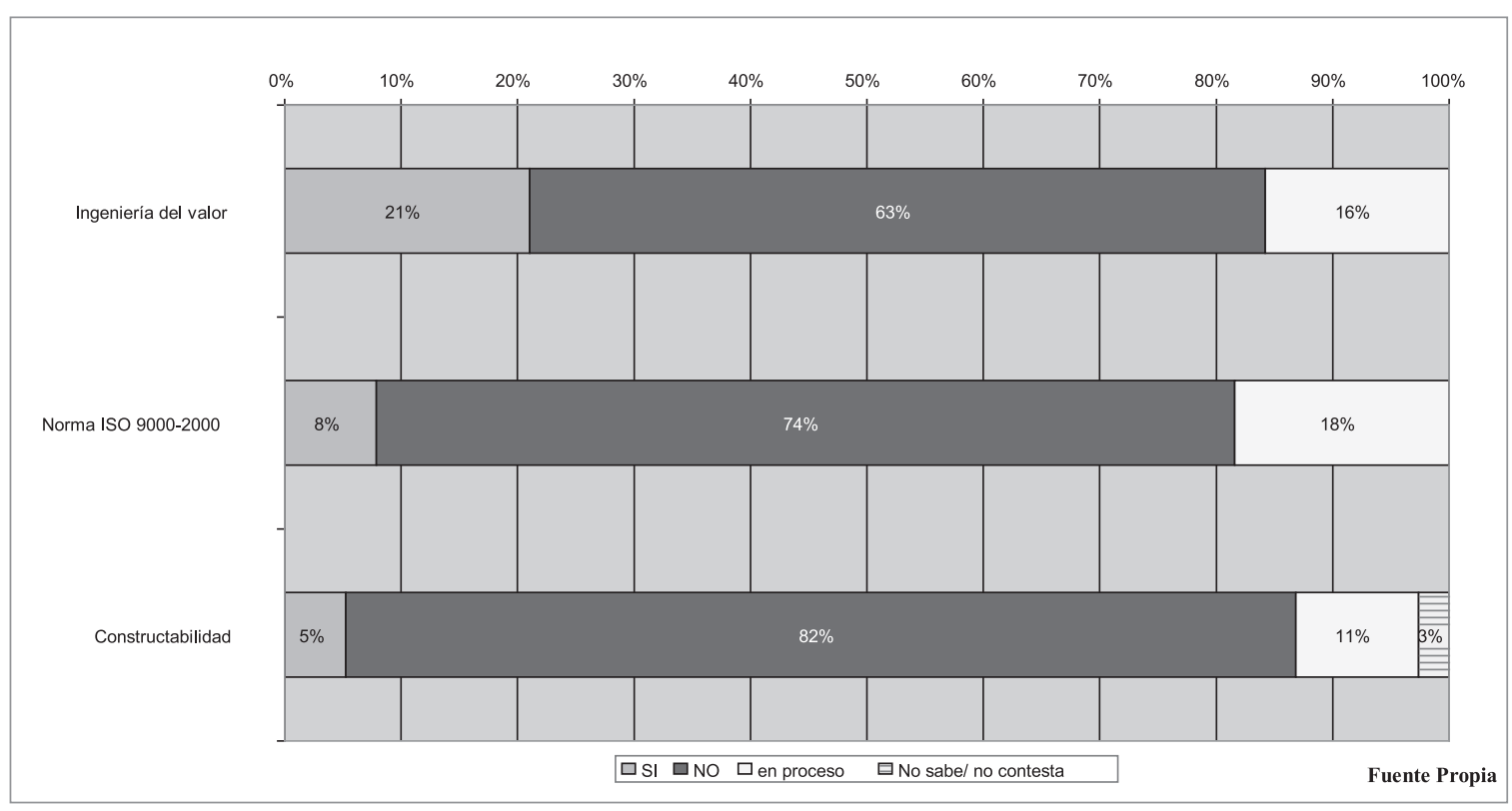

Figura 7. ¿Su empresa aplica/utiliza los siguientes proceso? 


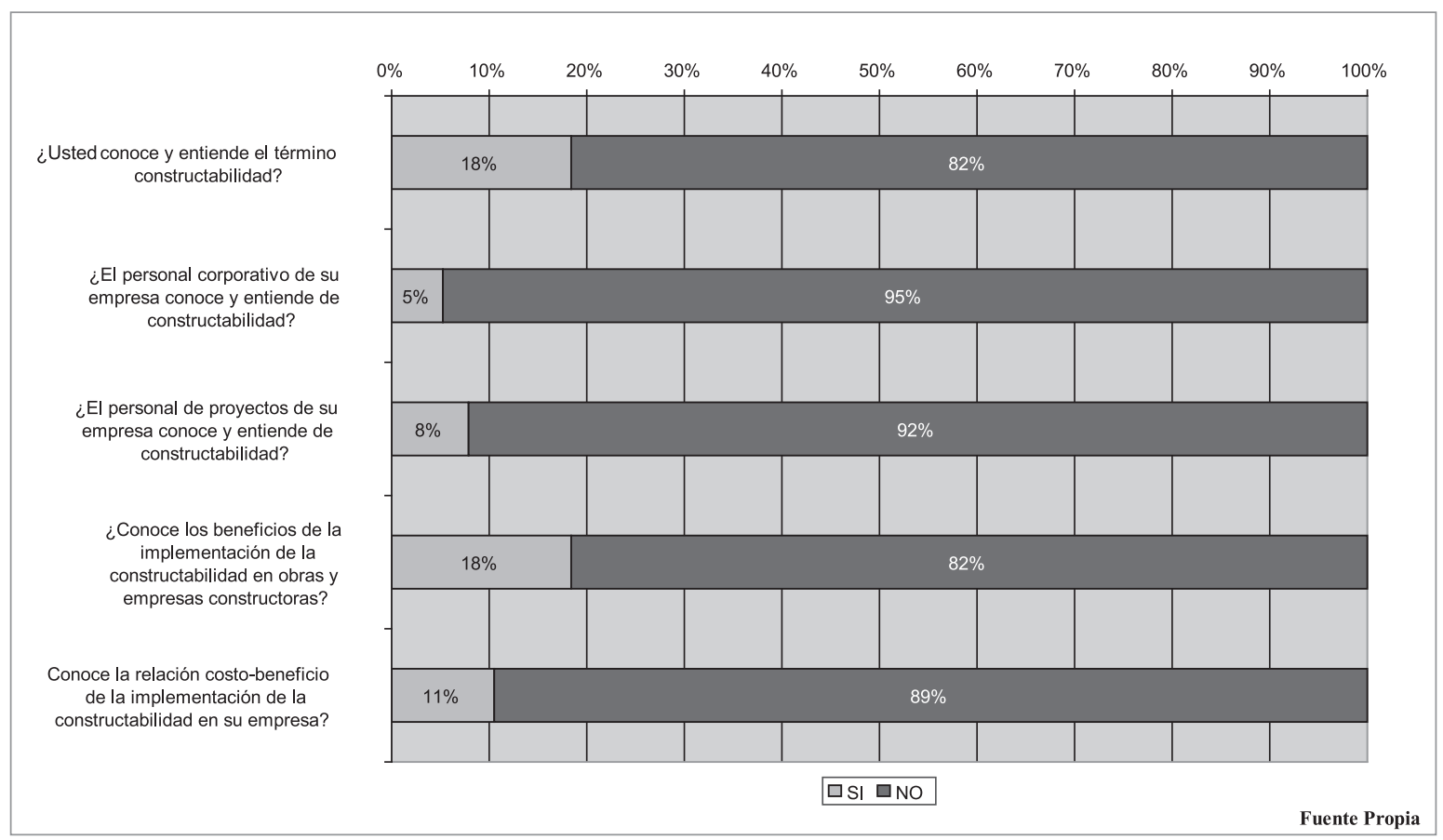

Figura 8. Conocimiento del programa de constructabilidad

Sin embargo la Figura 9 es muy significativa, ya que sin importar el hecho de que la mayoría de las empresas no tengan conocimiento sobre la constructabilidad, la disposición a su implementación es muy alta. Q uiere decir que hay una necesidad extendida de generar cambios para mejorar la productividad de los procesos internos de las empresas. Ejecutar cambios trascendentales en una empresa no es fácil, requiere de tiempo y dedicación, sin embargo los empresarios, manifiestan estar dispuestos a llevarlos a cabo en pro de optimizar su productividad, efectividad, eficiencia, rentabilidad, en fin mejorar la gestión de la construcción de la empresa.

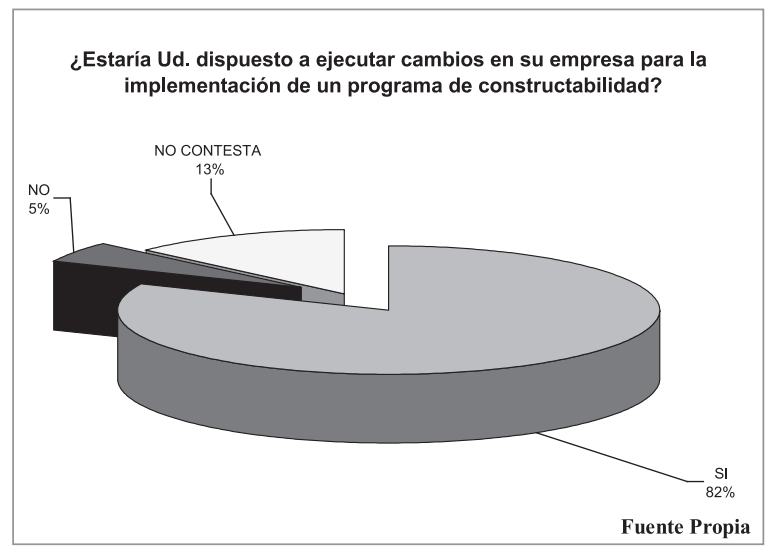

Figura 9. Disposición a la implementación del programa

\section{Conclusiones y recomendaciones}

De acuerdo con los resultados emitidos de la aplicación del cuestionario y tomando en consideración la situación actual de las empresas de obras civiles de la ciudad de Barquisimeto en cuanto al uso y conocimiento de la constructabilidad se concluye que:

La mayoría de las empresas desconoce el término "Constructabilidad", por esa razón, la implementación de la misma es nula en la ciudad. Sin embargo, las empresas se mostraron dispuestas a generar cambios para la implementación de este programa, siempre y cuando ayude a optimizar los procesos internos y de ejecución de las obras, mejore el rendimiento de los recursos, genere beneficios económicos, para así implementar donde sea más efectivo el programa, tanto en la etapa de diseño, procura y ejecución de la obra. Q uiere decir que hay una necesidad extendida de generar cambios para mejorar la productividad de los procesos internos de las empresas, bien sea utilizando la constructabilidad o cualquier otro programa de mejoramiento continuo que les aporte alguna solución a sus necesidades. 
Las empresas presentan, como era de esperarse, barreras para la implementación de la constructabilidad, esto indica que las empresas de la ciudad presentan inhibidores que evitan de facto la implementación efectiva del programa de constructabilidad en las organizaciones. Es por ello que debe atacarse en primer lugar los síntomas mas frecuentes, tales como la falta de documentación de errores cometidos y sus posibles correcciones, falta de visión de benchmarking, poco tiempo para el pensamiento estratégico y la falta de revisión del proyecto durante el proceso de diseño por parte del personal de construcción.

En cuanto al comportamiento de las empresas según los conceptos de constructabilidad, tenemos que éstas a pesar de no poseer conocimiento de la existencia de los mismos, los han implementado parcialmente de manera informal, como consecuencia de experiencia previa del personal, aunque sin la documentación debida y el seguimiento apropiado. En el caso específico de las consideraciones tempranas, las empresas se muestran preventivas en cuanto a algunos aspectos como por ejemplo, la selección de los métodos constructivos y revisión de las especificaciones de diseño, entre otras, así como también un gran porcentaje realiza programación de obras. Sin embargo en cuanto a la disponibilidad de materiales, equipos o mano de obra capacitada no están siendo efectivos, lo cual hace que no puedan evitar de manera eficaz ciertas dificultades en las obras, como por ejemplo los inconvenientes de procura y/o modificaciones durante la ejecución.

Ante la baja productividad presentada en el sector construcción (Serpell, 2002), se recomienda la implementación de los conceptos de constructabilidad, ya que éstos han demostrado en investigaciones previas de la CII (Preview of Constructability implementation, 1993), reducción de los excesos en costos por concepto de errores, y reducción de los retrasos en porcentajes importantes.

Se recomienda profundizar la investigación en los diversos aspectos relacionados con la gestión de la construcción y la implementación del programa de constructabilidad a raíz de este trabajo, como por ejemplo: (1) Ahondar en la severidad y en las causas de las barreras y problemas para la implementación de la constructabilidad. (2)Investigar las razones por las cuales las obras no se construyen exactamente igual al diseño original, para así determinar los correctivos necesarios a dicha problemática. (3)Medición de los porcentajes de trabajos productivos, contributorios y no contributorios y del fenómeno de aprendizaje de las empresas, con el fin de establecer metas certeras para el mejoramiento de la productividad en obras. (4) Indagar en las razones por las cuales existen interrupciones de las actividades y de las obras en general; así como también de las causas por las cuales las programaciones de las obras no son efectivas. (5) Evaluar las razones por las cuales se presentan dificultades en las obras, para así determinar las acciones correctivas que favorezcan la productividad y la reducción de tiempos y costos en obra.

Debido al alto grado de desconocimiento de la existencia del programa de constructabilidad y sus beneficios en costos y tiempos, se recomienda al sector académico generar un plan de capacitación permanente que beneficie a toda la comunidad con fines de mejorar la gestión de la construcción de las empresas de obras civiles de la región.

Se recomienda la creación de un comité de constructabilidad dentro de las instalaciones del decanato de ingeniería civil de la Universidad Centroccidental Lisandro Alvarado (UCLA) con la participación activa del sector productivo, con el fin de trabajar en conjunto para el mejoramiento de la gestión de la construcción de las distintas empresas y profesionales relacionados con el sector de la construcción que laboran en la región y en el país, tomando como referencia latinoamericana la experiencia de GEPUC (Centro de Excelencia en Gestión de Producción de la Pontificia Universidad de Chile). Aunque podrían incorporarse otras instituciones universitarias, se cree que la sede debe estar en el nombrado decanato, ya que es el único en la región que ofrece la carrera de ingeniería civil y la maestría de gerencia de la construcción. 


\section{Anexos}

Anexo 1. Ejemplo de las valoraciones en el cuestionario

\begin{tabular}{|c|c|c|c|c|c|c|}
\hline \multicolumn{7}{|c|}{ SECCION VIII. DIFICULTADES DE LA OBRA } \\
\hline & \multirow{2}{*}{$\begin{array}{l}\text { ¿Con qué frecuencia se le ha presentado las } \\
\text { siguientes situaciones? }\end{array}$} & SIEMPRE & $\begin{array}{l}\text { CASI } \\
\text { SIIEMPRE }\end{array}$ & $\begin{array}{l}\text { RLGUNAS } \\
\text { VECES }\end{array}$ & $\frac{\text { RARA }}{\text { VEZ- }}$ & NUNCA \\
\hline & & 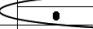 & 1 & 2 & 3 & 2 \\
\hline a & Inconvenientes con el diseño & $\square$ & 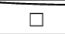 & $\square$ & 7 & $\square$ \\
\hline b & Ausencia de proyecto & $\square$ & $\square$ & & G & $\square$ \\
\hline & & $\begin{array}{l}\text { "Nunca" es } \\
\text { optima, por } \\
\text { la mayor va }\end{array}$ & $\begin{array}{l}\text { la respu } \\
\text { lo que t } \\
\text { loración }\end{array}$ & & & \\
\hline
\end{tabular}

Anexo 2. Parte del cuestionario utilizado para obtención de la información

\section{CUESTIONARIO SOBRE GESTION DE LA CONSTRUCCION} INSTRUCCIONES

El objetivo de este cuestionario es conocer la opinión de un grupo de gerentes y dueños de empresas, seleccionados por su destacada trayectoria en el sector construcción, sobre diversas variables que inciden en la gestión de la construcción.

El cuestionario consta de 10 secciones bajo el sistema de selección simple, en las cuales debe marcar con una $X$ o cualquier marca inequivoca la respuesta que considere al planteamiento a o la pregunta dada. No existen respuestas correctas 0 incorrectas io imporante es que responda de maner libre y directa En caso de marcar erróneamente una importante es que responda de manera libre y directa. En

Es imprescindible para los fines de la investigación que las respuestas se basen en la realidad actual de su empresa y no tomando en cuenta lo que desee o piense como el deber ser de la misma.

Es importante destacar que el cuestionario es estrictamente anónimo, es decir, no debe colocar su nombre, cargo o empresa encuestada. Sólo se le solicitará cierta información de la empresa con fines estadísticos. La información suministrada será manejada en estricta confidencialidad, analizada en conjunto y utilizada solo con fines académicos.

Cualquier duda puede consultar al administrador del cuestionario.

Gracias por dedicarle su valioso tiempo a la aplicación de este instrumento de investigación. Arq. Zulay Giménez Palavicin

Investigador Posigrado en Gerencia de la Construcción-DIC-UCLA.

CODIGO DE LA EMPRESA $\quad$ FECHA

Años de experiencia de la empresa

¿Pertenece a algún grupo de empresas filiales? $\quad$ Si $\square \quad$ No $\square$

Ciudad (es) donde opera:

Tipo de servicios prestados por la empresa:

$\square$ Proyectos de Diseño y Cálculos

$\square$ Promoción Inmobiliaria

$\square$ Construcción de Edificaciones

$\square$ Construcción de Obras Viales

$\square$ Construcción de Obras Sanitarias e Hidráulicas

$\square$ Otros

Porcentaje de contrataciones de su empresa

Contrataciones de obras públicas

Contrataciones de obras privadas $\%$

SECCION I. IDENTIFICACION DE BARRERAS DE IMPLEMENTACION

\begin{tabular}{|c|c|c|c|c|c|c|}
\hline \multicolumn{7}{|c|}{ SECCION I. IDENTIFICACION DE BARRERAS DE IMPLEMENTACION } \\
\hline & & SIEMPRE & $\begin{array}{c}\text { CASI } \\
\text { SIEMPRE }\end{array}$ & $\begin{array}{c}\text { RLGUNAS } \\
\text { VEGES }\end{array}$ & $\begin{array}{c}\text { RARR } \\
\text { VEZ }\end{array}$ & NUNCA \\
\hline & & 4 & 3 & 2 & 1 & - \\
\hline 1 & $\begin{array}{l}\text { ¿Tiene usted interés en implementar nuevas } \\
\text { estrategias en su empresa? }\end{array}$ & $\square$ & $\square$ & $\square$ & $\square$ & $\square$ \\
\hline 2 & $\begin{array}{l}\text { ¿Su empresa se toma un tiempo adecuado para } \\
\text { el pensamiento estratégico? }\end{array}$ & $\square$ & $\square$ & $\square$ & $\square$ & $\square$ \\
\hline 3 & $\begin{array}{l}\text { ¿El personal de diseño contratado por su } \\
\text { empresa tiene conocimiento de la construcción? }\end{array}$ & $\square$ & $\square$ & $\square$ & $\square$ & $\square$ \\
\hline 4 & $\begin{array}{l}\text { ¿El personal de construcción contratado por su } \\
\text { empresa revisa el proyecto durante el proceso } \\
\text { de diseño? }\end{array}$ & $\square$ & $\square$ & $\square$ & $\square$ & $\square$ \\
\hline 5 & $\begin{array}{l}\text { ¿El personal de su empresa interactúa } \\
\text { respetuosa y amablemente? }\end{array}$ & $\square$ & $\square$ & $\square$ & $\square$ & $\square$ \\
\hline 6 & $\begin{array}{l}\text { ¿Considera usted que su personal esta motivado } \\
\text { a buscar nuevos enfoques e ideas para resolver } \\
\text { problemas? }\end{array}$ & $\square$ & $\square$ & $\square$ & $\square$ & $\square$ \\
\hline 7 & $\begin{array}{l}\text { ¿Se evalúan los errores cometidos en las } \\
\text { obras? }\end{array}$ & $\square$ & $\square$ & $\square$ & $\square$ & $\square$ \\
\hline 8 & $\begin{array}{l}\text { ¿Se documentan dichos errores con su } \\
\text { respectiva posible solución? }\end{array}$ & $\square$ & $\square$ & $\square$ & $\square$ & $\square$ \\
\hline 9 & $\begin{array}{l}\text { ¿Es posible aumentar la eficacia en su } \\
\text { Empresa? }\end{array}$ & $\square$ & $\square$ & $\square$ & $\square$ & $\square$ \\
\hline 10 & $\begin{array}{l}\text { ¿Generalmente compara su empresa con } \\
\text { empresas líderes del área de la construcción? }\end{array}$ & $\square$ & $\square$ & $\square$ & $\square$ & $\square$ \\
\hline 11 & $\begin{array}{l}\text { ¿Se presentan las ideas de proyectos de } \\
\text { manera fácil de entender y no conflictiva? }\end{array}$ & $\square$ & 口 & $\square$ & $\square$ & $\square$ \\
\hline
\end{tabular}




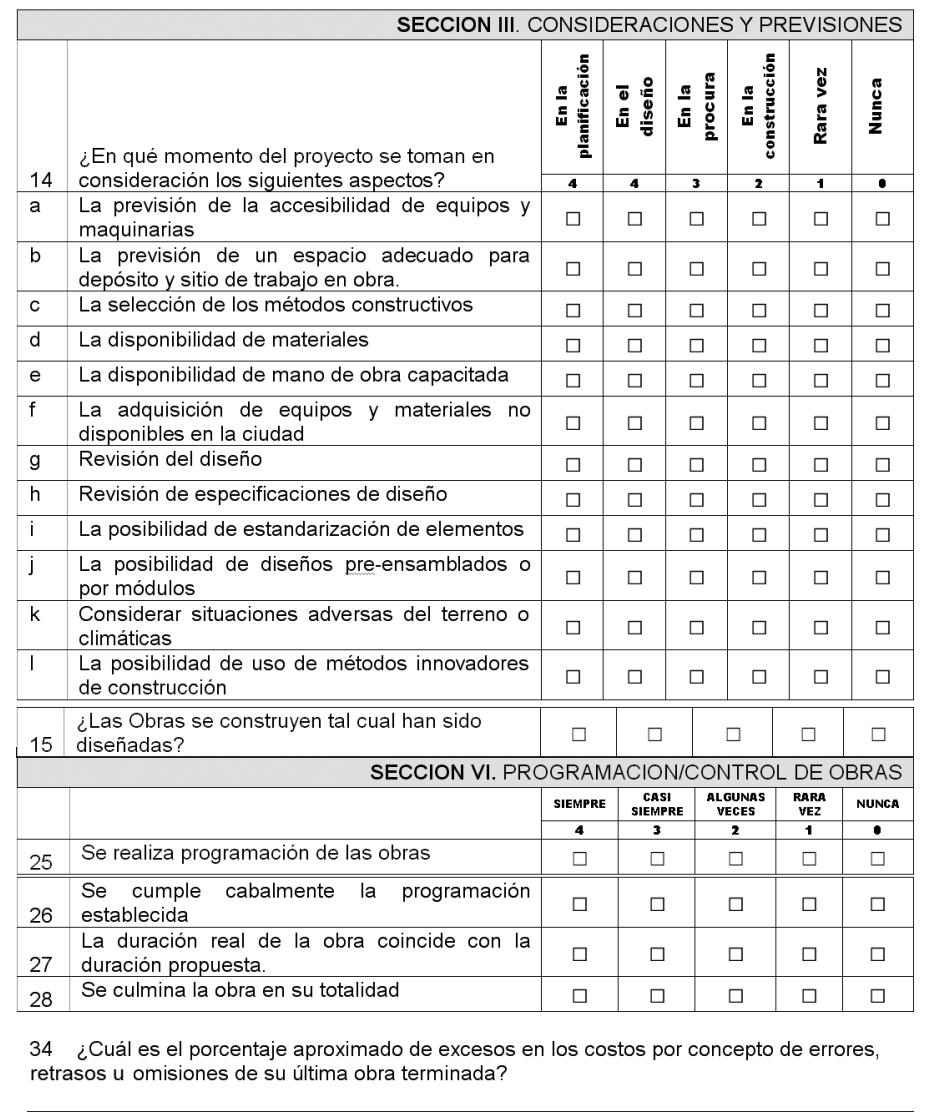

SECCION VIII. DIFICULTADES DE LA OBRA

\begin{tabular}{|c|c|c|c|c|c|c|}
\hline \multicolumn{7}{|c|}{ SECCION VIII. DIFICULTADES DE LA OBRA } \\
\hline & ¿Con qué frecuencia se le ha presentado las & SIEMPRE & $\begin{array}{c}\text { CASI } \\
\text { SIEMPRE }\end{array}$ & $\begin{array}{l}\text { RLGUNAS } \\
\text { VECES }\end{array}$ & $\begin{array}{c}\text { RARA } \\
\text { VEz }\end{array}$ & NUNCA \\
\hline 39 & siguientes situaciones? & - & 1 & 2 & 3 & 4 \\
\hline a & Inconvenientes con el diseño & $\square$ & $\square$ & $\square$ & $\square$ & $\square$ \\
\hline $\mathrm{b}$ & Ausencia de proyecto & $\square$ & $\square$ & $\square$ & $\square$ & $\square$ \\
\hline c & $\begin{array}{l}\text { Dificultades con los estudios previos } \\
\text { (topografia, replanteo, estudio de suelos) }\end{array}$ & $\square$ & $\square$ & $\square$ & $\square$ & $\square$ \\
\hline$d$ & Falta de Ingenieria de Detalles & $\square$ & $\square$ & $\square$ & $\square$ & $\square$ \\
\hline e & Contrariedades con los permisos & $\square$ & $\square$ & $\square$ & $\square$ & $\square$ \\
\hline$f$ & $\begin{array}{l}\text { Problemas legales (Expropiaciones, permisos } \\
\text { de paso, etc.) }\end{array}$ & $\square$ & $\square$ & $\square$ & $\square$ & $\square$ \\
\hline g & Dificultades de financiamiento & $\square$ & $\square$ & $\square$ & $\square$ & $\square$ \\
\hline $\mathrm{h}$ & $\begin{array}{l}\text { Problemas con equipos, maquinarias, } \\
\text { herramientas, etc. }\end{array}$ & $\square$ & $\square$ & $\square$ & $\square$ & $\square$ \\
\hline $\mathrm{i}$ & Modificaciones durante la ejecución & $\square$ & $\square$ & $\square$ & $\square$ & $\square$ \\
\hline j & Re-trabajo (Trabajos re-hechos) & $\square$ & $\square$ & $\square$ & $\square$ & $\square$ \\
\hline $\mathrm{k}$ & Entorpecimientos/exigencias de la comunidad & $\square$ & $\square$ & $\square$ & $\square$ & $\square$ \\
\hline I & Problemas con el clima & $\square$ & $\square$ & $\square$ & $\square$ & $\square$ \\
\hline $\mathrm{m}$ & Inconvenientes de procura & $\square$ & $\square$ & $\square$ & $\square$ & $\square$ \\
\hline $\mathrm{n}$ & Falta de inspección del trabajo & $\square$ & $\square$ & $\square$ & $\square$ & $\square$ \\
\hline 0 & Accidentes en el trabajo & $\square$ & $\square$ & $\square$ & $\square$ & $\square$ \\
\hline$p$ & $\begin{array}{l}\text { Complicaciones con el terreno(nivel freático, } \\
\text { grandes desniveles, accesibilidad) }\end{array}$ & $\square$ & $\square$ & $\square$ & $\square$ & $\square$ \\
\hline
\end{tabular}

SECCION IX. CONOCIMIENTO DEL PROGRAMA

\begin{tabular}{|c|c|c|c|c|}
\hline \multicolumn{5}{|c|}{ SECCION IX. CONOCIMIENTO DEL PROGRAMA } \\
\hline & & sI & No & En \\
\hline 40 & Su empresa aplica/utiliza los siguientes procesos & 4 & $\bullet$ & 2 \\
\hline a & Ingeniería del valor & $\square$ & $\square$ & $\square$ \\
\hline b & Norma ISO $9000-2000$ & $\square$ & $\square$ & $\square$ \\
\hline$c$ & Constructabilidad & $\square$ & $\square$ & $\square$ \\
\hline 41 & ¿Usted conoce y entiende el término constructabilidad? & $\square$ & $\square$ & \\
\hline 42 & $\begin{array}{l}\text { ¿El personal corporativo de su empresa conoce y entiende de } \\
\text { constructabilidad? }\end{array}$ & $\square$ & $\square$ & \\
\hline 43 & $\begin{array}{l}\text { ¿El personal de proyectos de su empresa conoce y entiende de } \\
\text { constructabilidad? }\end{array}$ & $\square$ & $\square$ & \\
\hline 44 & $\begin{array}{l}\text { ¿Conoce los beneficios de la implementación de la } \\
\text { constructabilidad en obras y empresas constructoras? }\end{array}$ & $\square$ & $\square$ & \\
\hline 45 & $\begin{array}{l}\text { ¿Conoce la relación costo-beneficio de la implementación de la } \\
\text { constructabilidad en su empresa? }\end{array}$ & $\square$ & $\square$ & \\
\hline
\end{tabular}

45 constructabilidad en su empresa?

SECCION X. DISPOSICION A LA IMPLEMENTACIÓN DEL PROGRAMA

¿Estaría usted dispuesto a ejecutar cambios en su empresa para la

46 implementación de un programa de constructabilidad?

Especifique: 
Anexo 3

Tabla 10. Relación de pregunta con síntoma de barrera/problema de implementación

\begin{tabular}{|c|c|}
\hline Pregunta del cuestionario & Síntoma de barrera/problema \\
\hline $\begin{array}{l}\text { ¿Tiene usted interés en implementar nuevas } \\
\text { estrategias en su empresa? }\end{array}$ & $\begin{array}{r}\text { Poco interés en la implementación de nuevas } \\
\text { estrategias }\end{array}$ \\
\hline $\begin{array}{l}\text { ¿Su empresa se toma un tiempo adecuado para } \\
\text { el pensamiento estratégico? }\end{array}$ & Poco tiempo para el pensamiento estratégico \\
\hline $\begin{array}{l}\text { ¿El personal de diseño contratado por su } \\
\text { empresa tiene conocimiento de la } \\
\text { construcción? }\end{array}$ & $\begin{array}{r}\text { El personal de diseño contratado por la } \\
\text { empresa no tiene conocimiento de la } \\
\text { construcción }\end{array}$ \\
\hline $\begin{array}{l}\text { ¿El personal de construcción contratado por su } \\
\text { empresa revisa el proyecto durante el proceso } \\
\text { de diseño? }\end{array}$ & $\begin{array}{l}\text { El personal de construcción no revisa el } \\
\text { proyecto durante el proceso de diseño }\end{array}$ \\
\hline $\begin{array}{l}\text { ¿El personal de su empresa interactúa } \\
\text { respetuosa y amablemente? }\end{array}$ & $\begin{array}{r}\text { Problemas de relaciones personales entre los } \\
\text { empleados de la empresa }\end{array}$ \\
\hline $\begin{array}{l}\text { ¿Considera usted que su personal esta motivado } \\
\text { a buscar nuevos enfoques e ideas para resolver } \\
\text { problemas? }\end{array}$ & $\begin{array}{r}\text { Falta de motivación a buscar innovaciones para } \\
\text { resolución de problemas }\end{array}$ \\
\hline ¿Se evalúan los errores cometidos en las obras? & Falta de evaluación de los errores cometidos \\
\hline $\begin{array}{l}\text { ¿Se documentan dichos errores con su } \\
\text { respectiva posible solución? }\end{array}$ & $\begin{array}{r}\text { Falta de documentación de errores cometidos y } \\
\text { sus posibles correcciones }\end{array}$ \\
\hline $\begin{array}{l}\text { ¿Es posible aumentar la eficacia en su } \\
\text { Empresa? }\end{array}$ & Complacencia con su estado actual \\
\hline $\begin{array}{l}\text { ¿Generalmente compara su empresa con } \\
\text { empresas líderes del área de la construcción? }\end{array}$ & $\begin{array}{l}\text { Falta de visión de benchmarking (No hay } \\
\text { comparación del desempeño) }\end{array}$ \\
\hline $\begin{array}{l}\text { ¿Se presentan las ideas de proyectos de manera } \\
\text { fácil de entender y no conflictiva? }\end{array}$ & $\begin{array}{r}\text { Problemas de comunicación entre áreas/Falta } \\
\text { de simplicidad }\end{array}$ \\
\hline
\end{tabular}

\section{Referencias}

Aim-andalucia (2006), http://www.aimandalucia.com/indexConstructividad.htm - febrero de 2006

Cámara de la Construcción del Estado Lara (2006), www.ccl.com.ve - abril de 2006

Construction Industry Institute (1993), Implementation guide Constructability. Publication 34-1. The U niversity of Texas, Austin. Mayo.

Construction Industry Institute (1993), Preview of Constructability implementation. Bureau of Engineering Research publication 34-2. The U niversity of Texas, Austin. February.

Hernandez Sampieri (2004), R. Metodología de la Investigación. McGraw-Hill. México.
M eans, M ethods and Trends (2006), http://w ww.mmtmagazine.org/page/index4018.html?i $\mathrm{d}=173$ - marzo de 2006

Pocock, James B. (2006), Constructability state of practice report. April .

Rischmoller L. (2006), Jornadas de Constructabilidad, Universidad Centroccidental Lisandro Alvarado, 27 de O ctubre.

Serpell, A. (2002), Administración de operaciones de construcción. Textos U niversitarios. Facultad de Ingeniería. Ediciones Universidad Católica de Chile. Chile.

Villalba, J. (1999), Menú Estratégico. El arte de la guerra competitiva. Ediciones IESA. Caracas, Venezuela.

Walpole, R. y Myers, R. (2000), Probabilidad y Estadística. McGraw-Hill. Cuarta edición. México. 